\title{
ÉTUDE DE LA STABILITÉ TEMPORELLE DES STRUCTURES SPATIALES PAR ANALYSES D'UNE SÉRIE DE TABLEAUX DE RELEVÉS FAUNISTIQUES TOTALEMENT APPARIÉS.
}

\author{
L. BLANC (1), D. CHESSEL (2), S. DOLÉDEC (2)
}

(1) UMR - CNRS 5556, Laboratoire d'Hydrobiologie Marine et Continentale, case 093, Université Montpellier II, place Eugène Bataillon, 34095 Montpellier Cedex 5, France.

(2) ESA - CNRS 5023, Laboratoire d'Ecologie des Eaux Douces et des Grands Fleuves, Université Lyon I, Bat. 401C, 43 boulevard du 11 novembre 1918, 69622 Villeurbanne Cedex, France.

\section{RÉSUMÉ}

L'analyse triadique partielle (ATP), introduite en écologie par THIOULOUSE et CHESSEL (1987), est reprise dans cet article. Le principe de la méthode est présenté de façon simplifiée afin de faciliter son usage. Cette méthode permet de faire les analyses en composantes principales (ACP) simultanées et coordonnées de plusieurs tableaux de relevés faunistiques ou mésologiques effectués, par exemple, dans différentes localités de façon répétée dans le temps. Dans ce cas, l'ATP permet de trouver une structure spatiale stationnelle commune à tous les tableaux (dates d'échantillonnage) et d'en étudier la stabilité temporelle. Pour cela, la méthode s'appuie sur la recherche d'une moyenne de tableaux appelée compromis et sur la reproductibilité du compromis par chacun des tableaux initiaux. Nous présentons également l'analyse d'une série de tables de contingence (FOUCART, 1978) qui, dans le même contexte expérimental, permet d'utiliser une stratégie d'analyse factorielle des correspondances (AFC) plutôt qu'une stratégie d'ACP. Nous illustrons le potentiel de ces méthodes multitableaux à l'aide d'un exemple en hydrobiologie en soulignant leur capacité à décrire une évolution temporelle de structures spatiales par rapport à une référence commune.

Mots-clés : écologie statistique, analyse triadique partielle, AFC de FOUCART, série de tables de contingence, compromis, stabilité, espace, temps, structure des communautés.

\section{STUDY OF THE TEMPORAL STABILITY OF SPATIAL STRUCTURES BY ANALYSING SERIES OF TOTALLY PAIRED FAUNISTIC TABLES.}

\section{ABSTRACT}

In this paper, we investigate the potential of partial triadic analysis (PTA), already introduced in ecology by THIOULOUSE and CHESSEL (1987). The principle of the method is presented in a simplified way to facilitate its use by ecologists. This method allows the simultaneous principal components analyses (PCA) of several species composition tables corresponding to the collection of organisms in various locations at different occasions to be 
coordinated. In this case, PTA permits to find a spatial structure common to every table (i.e., sampling occasion) and to study its temporal stability. Therefore, PTA starts by searching an average table called compromise. Then, the compromise table is analysed and its reproductibility by each initial table is finally investigated. Using the same sampling design, we also present the analysis of a series of contingency tables (FOUCART, 1978) which represents a different approach since it considers the frequency distribution of species rather than their abundance. We illustrate these two K-set methods by an example in stream ecology. We demonstrate the power of these methods for depicting the temporal evolution of the spatial structures.

Key-words : statistical ecology, partial triadic analysis, FOUCART's analysis, series of contingency tables, compromise, stability, spatial, temporal, community structure.

\section{INTRODUCTION}

On ne peut rigoureusement appréhender la structure d'un système qu'en tenant compte de sa variabilité dans le temps. II est donc fréquent que le système étudié fasse l'objet d'un échantillonnage spatial (quadrats, transects) de plusieurs stations de façon répétée dans le temps. On obtient alors des tableaux à trois indices : (1) la station, (2) le descripteur, c'est-à-dire la variable mesurée (biotique ou abiotique), et (3) la date d'échantillonnage. De tels plans d'échantillonnage sont appliqués par exemple pour étudier la variabilité spatio-temporelle des peuplements. Ils permettent ainsi d'approcher la dynamique d'un peuplement faunistique (CARREL et al., 1995) ou d'évaluer la stabilité dans le temps de sa répartition spatiale (LETOURNEUR et CHABANET, 1994). Un des objectifs principaux de l'écologie des communautés est alors de mettre en relation la structure spatio-temporelle des variables abiotiques et biotiques mesurées sur les différentes localités échantillonnées. De nombreuses études ont été réalisées dans ce but dans des environnements variés et portant par exemple sur l'ichtyofaune des milieux lagunaires ou estuariens (ALIAUME et al., 1993 ; BARAN, 1995 ; HERREIRA-SILVEIRA, 1994 ; MEES et al., 1995), des rivières (HAURY et al., 1995) ou des lacs (DEGIORGI, 1994), sur les peuplements de juvéniles de poissons en milieu fluvial (POIZAT et PONT, 1996 ; PENAZ et al., 1995). Les communautés de macroinvertébrés benthiques de grands fleuves (DOLÉDEC et al., 1996), les communautés phytoplanctoniques (IZAGUIRRE et VINOCUR, 1994) ou zooplanctoniques (AMBLARD et al., 1995) de lacs font également l'objet de plans d'échantillonnage spatio-temporels. Ces études aboutissent à l'élaboration de typologies spatiales ou temporelles moyennes.

L'usage des méthodes d'analyses multivariées classiques telles que l'analyse en composantes principales (ACP), l'analyse factorielle des correspondances (AFC) ou l'analyse des correspondances multiples $(A C M)$, est désormais très répandu pour traiter des tableaux de relevés écologiques de type stations-variables environnementales (CARREL et al., 1986 ; BAGLINIĖRE et al., 1987 ; MUSSO et al., 1991), stations-relevés faunistiques (VERNEAUX, 1973 ; BOET et al., 1991; CHANGEUX, 1995) ou stationsrelevés floristiques (GRASMÜCK et al., 1995 ; COSTIL et CLEMENT, 1996).

Des outils statistiques appropriés à l'analyse de ce type de données ont été développés par exemple en hydrobiologie. DOLÉDEC et CHESSEL $(1987,1989)$ ont ainsi proposé des méthodes dites inter- et intra-classes qui utilisent le temps et l'espace comme de véritables variables instrumentales contrôlant l'analyse. Ces méthodes d'ordination sous contraintes dont on a une revue dans BIRKS et al. (1994) permettent en première approche d'étudier l'effet spatial (stations) ou l'effet temporel (dates) et de mesurer l'interaction datesstations dans des tableaux faunistiques ou mésologiques (BEFFY et DOLÉDEC, 1991 ; DEGIORGI et GRANDMOTTET, 1993 ; LAIR et SARGOS, 1993 ; BORNETTE et al., 1994 ; FRANQUET et al., 1995). 
Les principes de l'analyse triadique partielle (JAFFRENOU, 1978 ; THIOULOUSE et CHESSEL, 1987 ; KROONENBERG, 1989) et de l'AFC de FOUCART $(1978,1983)$ présentés ici sont différents. Contrairement aux analyses inter- et intra-classes, ces méthodes multitableaux ne cherchent pas à décomposer la variabilité des mesures selon l'espace ou le temps mais à décrire directement les structures induites dans les données par l'organisation du plan d'échantillonnage spatio-temporel. Les méthodes présentées ici s'appliquent au cas où les tableaux ont le même nombre de lignes et le même nombre de colonnes. Elles permettent de définir la structure commune à ces tableaux (méthodes pour traiter les « three-way data arrays ", BOVE et DI CIACCIO, 1994). Quelques rares exemples d'utilisation (AMANIEU et al., 1981; DOLÉDEC, 1988 ; CENTOFANTI et al., 1989 ; ALIAUME et al., 1993) soulignent la difficulté d'utilisation de ces méthodes par les écologistes malgré les perspectives d'approches fonctionnelles des typologies qu'elles permettent.

Le but de cet article est d'illustrer ces deux méthodes multitableaux de base par un exemple d'application à des communautés d'invertébrés aquatiques d'eaux courantes en simplifiant l'approche adoptée initialement par THIOULOUSE et CHESSEL (1987) et en développant les aides à l'interprétation.

\section{MATÉRIEL ET MÉTHODES}

\section{Principe de l'analyse triadique partielle}

La situation dans laquelle nous allons nous placer est reprise dans la Figure 1A. Comme nous l'avons déjà indiqué, l'usage de l'analyse triadique partielle impose d'utiliser les mêmes variables mesurées sur les mêmes stations à plusieurs reprises. Chaque élément de tableau $\mathrm{x}_{\mathrm{ijk}}$ appartient à un tableau rectangulaire (station-ligne $i \mathrm{x}$ variablecolonne $f$ ) correspondant à une date $k$. Nous avons considéré les données selon une suite chronologique de tableaux [stations $x$ variables], l'objectif étant de faire un bilan des structures spatiales et de leur stabilité (Figure 1A). On pourrait aborder le problème autrement en considérant une suite de tableaux [dates $x$ variables] avec un tableau par station. L'objectif serait alors différent puisque l'on rechercherait à faire un bilan des évolutions temporelles et de leur répartition dans l'espace, mais l'approche statistique serait la même.

L'analyse triadique partielle appartient à la "famille" des méthodes STATIS (L'HERMIER DES PLANTES, 1976 ; LAVIT, 1988 ; LAVIT et al., 1994) qui permettent l'analyse conjointe de plusieurs tableaux. Le principe général est de trouver à partir de ces tableaux une structure (ou typologie) moyenne qui servira de base à la comparaison des tableaux entre eux. Dans le cas où les tableaux n'ont en commun que le nombre de lignes ou de colonnes, its ne sont pas directement comparables et on utilise alors la méthode STATIS proprement dite. Quand les nombres de lignes et de colonnes sont identiques, on utilise l'analyse triadique partielle, dite encore STATIS sur les X (LEIBOVICI, 1993) et on peut calculer directement un tableau " compromis " correspondant à une somme pondérée des $\mathrm{K}$ tableaux initiaux.

Soit $K$ tableaux ayant $n$ lignes et $p$ colonnes chacun. A l'intersection de la ligne $i$ et de la colonne $j$ on trouve la valeur de la variable $j$ mesurée à la station $i$. Après transformations initiales (centrage, normalisation, etc.) on obtient $K$ tableaux $\mathbf{X}_{k}$ correspondant aux $k$ dates avec le même nombre de lignes et de colonnes et ayant pour éléments $x_{i, j}^{k}$. En s'appuyant sur le schéma de dualité (ESCOUFIER, 1987), on peut définir pour chacun des tableaux le triplet $\left(\mathbf{X}_{k}, \mathbf{D}_{p}, \mathbf{D}_{n}\right)$ où $\mathbf{D}_{p}$ et $\mathbf{D}_{n}$ sont respectivement les matrices diagonales des poids des 


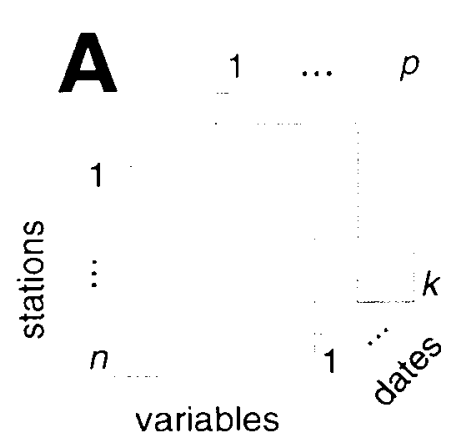

variables

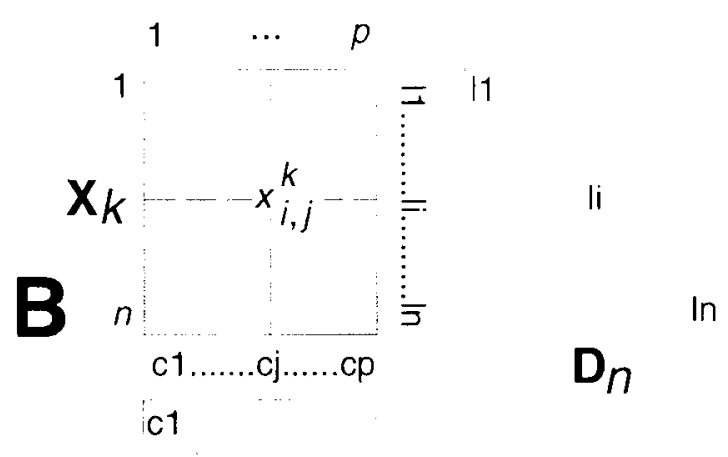

cj<smiles>[2H][O]</smiles>

Figure 1

(A) Plan expérimental. Les mêmes $p$ variables (abiotiques ou biotiques) ont été échantillonnées à chaque date $k$, et à chaque station $n$. (B) Un tableau $X_{k}$ est défini par le triplet statistique $\left(X_{k}, D_{p}, D_{n}\right)$ où $D_{p}$ et $D_{n}$ sont respectivement les matrices diagonales des poids des colonnes $(c 1 \ldots c p)$ et des poids des lignes $(11 \ldots \mid n)$.

\section{Figure 1}

(A) Sampling design. The same $p$ variables (abiotic or biotic) were sampled at each date $k$, and each station $n$. (B) The $X_{k}$ array is defined by the statistical triplet $\left(X_{k}, D_{p}, D_{n}\right)$ where $D_{p}$ is the column weight matrix $(c 1 \ldots c p)$ and $D_{n}$ is the row weight matrix $(11 \ldots . \mid n)$.

colonnes et des poids des lignes (Figure 1B). Pour comparer deux tableaux $\mathbf{X}_{k}$ et $\mathbf{X}_{l}$, on calcule un produit scalaire défini par ESCOUFIER (1973) comme leur covariance vectorielle (Covv):

$$
\operatorname{Covv}\left(\mathbf{X}_{k}, \mathbf{X}_{l}\right)=\operatorname{Trace}\left(\mathbf{X}_{k}^{t} \mathbf{D}_{n} \mathbf{X}, \mathbf{D}_{p}\right)=\operatorname{Trace}\left(\mathbf{X}_{i}^{t} \mathbf{D}_{n} \mathbf{X}_{k} \mathbf{D}_{p}\right)
$$

$\mathbf{x}_{k}^{t}$ étant la transposée de la matrice $\mathbf{x}_{k}$ et $\operatorname{Trace}(\mathbf{X})$ représentant la somme des termes diagonaux de la matrice $\mathbf{X}$. De la même façon, on calcule la variance vectorielle d'un tableau $\mathbf{X}_{k}\left(\operatorname{Vav}\left(\mathbf{X}_{k}\right)\right)$ :

$$
\operatorname{Vav}\left(\mathbf{X}_{k}\right)=\operatorname{Trace}\left(\mathbf{X}_{k}^{t} \mathbf{D}_{n} \mathbf{X}_{k} \mathbf{D}_{p}\right)
$$

d'où l'expression du coefficient de corrélation vectorielle entre deux tableaux :

$$
\operatorname{Rv}\left(\mathbf{X}_{k}, \mathbf{X}_{l}\right)=\frac{\operatorname{Covv}\left(\mathbf{X}_{k}, \mathbf{X}_{i}\right)}{\sqrt{\operatorname{Vav}\left(\mathbf{X}_{k}\right)} \sqrt{\operatorname{Vav}\left(\mathbf{X}_{l}\right)}}
$$

On peut considérer qu'un coefficient Rv est l'équivalent entre deux tableaux d'une corrélation entre deux variables. Un coefficient Covv est par contre l'équivalent entre deux tableaux d'une covariance entre deux variables. On peut ainsi établir une matrice de corrélations vectorielles inter-tableaux équivalent de la matrice des corrélations inter-variables 
en ACP normée ou une matrice de covariances vectorielles inter-tableaux équivalent de la matrice des covariances inter-variables en ACP centrée.

L'analyse triadique partielle se déroule alors classiquement en trois étapes décrites ci-après.

Première étape. Phase de l'interstructure et recherche du compromis. On recherche dans un premier temps une " moyenne " $X$ (ou compromis) des $K$ tableaux, somme pondérée de ces tableaux:

$$
\mathbf{X}=\sum_{k=1}^{K} \alpha_{k} \mathbf{X}_{k}
$$

Les coefficients $\alpha_{k}$ sont calculés tels que l'analyse du triplet $\left(X, \mathbf{D}_{p}, \mathbf{D}_{n}\right)$ soit optimale, c'est-à-dire qu'elle présente une inertie interne maximale sous la contrainte :

$$
\sum_{k=1}^{k} \alpha_{k}^{2}=1
$$

Ces coefficients sont les composantes normées du premier vecteur propre de la matrice des covariances vectorielles entre tableaux (Covv) obtenus après diagonalisation de cette matrice. On peut aussi diagonaliser la matrice des corrélations Rv, comme c'est le cas habituellement dans les analyses STATIS. Dans le cas de l'analyse triadique partielle, on préférera diagonaliser la matrice des Covv car on suppose logiquement que des tableaux totalement appariés (mêmes lignes et mêmes colonnes) présentent des inerties de même ordre de grandeur. Si ce n'est pas le cas, cette information intrinsèque aux données rentre en ligne de compte dans le calcul du compromis.

L'étape de diagonalisation de la matrice des Covv est appelée dans la terminologie de STATIS étape d'interstructure. A la différence de l'approche de THIOULOUSE et CHESSEL (1987), on ne s'étendra pas ici sur l'analyse de cette étape dont nous retenons seulement le premier vecteur propre qui a une fonction de définition d'une moyenne alors que les suivants caractérisent l'écart au modèle moyen. La diagonalisation a donc ici pour fonction essentielle d'attribuer à chaque tableau un poids (les composantes normées du premier vecteur propre). Un nouveau tableau de synthèse combinant les tableaux initiaux en proportion de leurs poids dans la description de la structure commune dite compromis est ainsi constitué. Le cosinus carré ( $\operatorname{Cos}^{2}$ entre le tableau $k$ et le compromis (c'est un Rv Cf. (3))) constitue un indicateur de la qualité de l'expression par un tableau donné de la structure du compromis.

Deuxième étape. Analyse du compromis. L'analyse du tableau compromis s'effectue comme une analyse d'inertie standard. Elle fournit des axes principaux et des composantes principales respectivement dans l'espace des lignes et dans l'espace des colonnes sur lesquels se projettent les colonnes et les lignes du tableau compromis. Cette analyse permet d'établir une typologie commune à tous les tableaux.

Troisième étape. Analyse de la reproductibilité du compromis. On peut finalement projeter sur les axes et composantes principales du compromis les lignes, les colonnes, les axes et les composantes principales des analyses séparées de chacun des tableaux. Ces opérations permettent de discuter de la stabilité des tableaux autour de la moyenne (compromis), autrement dit d'analyser la reproductibilité de la structure du compromis par chacun des tableaux. Pour cela, des aides à l'interprétation telles que les représentations graphiques multifenêtrées par tableau sont conseillées. 


\section{Analyse de séries de tableaux de contingence}

Face à un tableau de données faunistiques, on a principalement le choix entre deux méthodes d'analyses multivariées classiques: l'analyse factorielle des correspondances (AFC) où la transformation qui est effectuée correspond à un double centrage multiplicatif et l'analyse en composantes principales (ACP) centrée par espèce (chaque espèce a une moyenne nulle) (voir par exemple DOLÉDEC et CHESSEL, 1991). Quand on traite plusieurs tableaux faunistiques ayant le même nombre de lignes et de colonnes, l'analyse triadique partielle présentée ci-dessus permet de faire des ACP simultanées et coordonnées des tableaux. L'usage de l'AFC impose une pondération propre à chacun des tableaux et non identique d'un tableau à l'autre puisque les poids des lignes et des colonnes sont calculés avec les données. Or, l'analyse triadique partielle exige que tous les tableaux aient des pondérations égales en lignes et en colonnes. Pour lever ce problème, FOUCART (1978, 1983) part de la constatation que l'on peut aussi bien concevoir une table de contingence comme un tableau d'ACP particulière que comme une matrice de covariance particulière. L'auteur suggère donc de construire le compromis en prenant une moyenne uniformément pondérée des $K$ tableaux.

Soit $K$ tableaux d'AFC ; le $k^{\text {ème }}$ tableau a, comme les autres, $i$ lignes et $j$ colonnes. Son terme général est égal à $x_{i j}^{k}$ et la somme de toutes les valeurs est égale à $x^{k}$.. Le tableau de fréquences associé est égal à :

$$
\mathbf{P}_{k}=\left[x_{i j}^{k} / x^{k}\right]
$$

La moyenne est égale à :

$$
\mathbf{P}=(1 / K) \sum_{k} \mathbf{P}_{k}
$$

L'AFC de FOUCART va consister en l'analyse des correspondances du tableau moyen $\mathbf{P}$, structure compromis utilisant une pondération uniforme. L'intrastructure est réalisée en projetant en individus supplémentaires les lignes et les colonnes des $K$ tableaux de départ. Les pondérations de l'AFC du compromis servent de référence générale. Si l'on n'a aucune raison de pondérer inégalement les tableaux, cette analyse ne pose aucun problème de signification pour l'utilisateur. A notre connaissance, cette méthode n'a encore été que très peu appliquée en écologie (BARAN, 1995).

\section{Données}

Nous illustrons ces deux méthodes à l'aide d'un jeu de données constitué par PEGAZ-MAUCET (1980) et largement décrit par DOLÉDEC et CHESSEL (1991) et FRANQUET et al. (1995). L'auteur des données s'intéressait à l'impact d'une pollution organique sur le peuplement en macroinvertébrés benthiques d'un cours d'eau. Six stations ont été échantillonnées le long du Méaudret (affluent de la Bourne, Vercors) à quatre saisons (printemps, été, automne et hiver). La station 6, située sur la Bourne, n'est pas prise en compte ici.

A chaque saison et à chaque station, des relevés faunistiques concernant les communautés d'invertébrés ont été réalisés; parmi ceux-ci, nous avons retenu le groupe des Ephéméroptères composé de 13 taxons. Les données étudiées sont donc formées de quatre tableaux (un par saison) portant sur les cinq mêmes stations et les 13 mêmes descripteurs faunistiques (Tableau I). 


\section{Tableau I}

Données faunistiques traitées (d'après PEGAZ-MAUCET, 1980). Les quatre blocs de cinq lignes correspondent aux quatre dates et aux cinq stations échantillonnées. Les treize colonnes correspondent à treize taxons d'Ephéméroptères. Les données représentent des classes d'abondance de progression géométrique de raison 2.

Codes des espèces $:$ Eda $=$ Ephemera danica Müller, $1764 ;$ Bsp = Baetis sp. (Leach, 1815) ; Brh = Baetis rhodani (Pictet, 1843) ; Bni = Baetis niger (Linnaeus, 1761) ; Bmu = Baetis muticus (Linnaeus, 1758) ; Cen = Centroptilum sp. (Eaton, 1869) ; Ecd = Ecdyonorus sp. (Eaton, 1865) ; Rhi = Rhithrogena sp. (Eaton, 1881) ; Hla = Habrophlebia lauta Eaton, 1884 ; Hab = Habroleptoides sp. Schönemund, $1929 ;$ Par = Paraleptophlebia sp. $($ Lestage, 1917) $;$ Cae = Caenis sp. Stephens, $1835 ;$ Eig = Ephemerella ignita $($ Poda, 1761).

Table I

Species composition data (from PEGAZ-MAUCET, 1980). The four groups of five rows correspond to the four sampling dates and the five sampling stations. The thirteen Ephemeropteran taxa occur as columns. Data are transformed into $\log _{2}(x)$.

Species codes : Eda = Ephemera danica Müller, $1764 ;$ Bsp = Baetis sp. $($ Leach, 1815) ; Brh = Baetis rhodani (Pictet, 1843); Bni = Baetis niger (Linnaeus, 1761); Bmu = Baetis muticus (Linnaeus, 1758); Cen = Centroptilum sp. (Eaton, 1869); Ecd = Ecdyonorus sp. (Eaton, 1865) ; Rhi = Rhithrogena sp. (Eaton, 1881); $\mathrm{Hla}=$ Habrophlebia lauta Eaton, 1884 ; Hab = Habroleptoides sp. Schönemund, $1929 ;$ Par = Paraleptophlebia sp. $($ Lestage, 1917) $;$ Cae = Caenis sp. Stephens, 1835 ; Eig = Ephemerella ignita (Poda, 1761).

\begin{tabular}{|c|c|c|c|c|c|c|c|c|c|c|c|c|c|}
\hline Dates & $\overline{E d a}$ & Bsp & $\overline{\mathrm{Brh}}$ & Bni & $\mathrm{Bmu}$ & Cen & Ecd & Rhi & $\mathrm{Hla}$ & $\overline{\mathrm{Hab}}$ & Par & Cae & Eig \\
\hline & 4 & 7 & 10 & 9 & 0 & 0 & 0 & 5 & 9 & 0 & 4 & 0 & 0 \\
\hline & 0 & 0 & 8 & 0 & 0 & 0 & 0 & 0 & 4 & 0 & 0 & 0 & 0 \\
\hline Printemps & 0 & 5 & 5 & 0 & 0 & 0 & 0 & 2 & 5 & 0 & 0 & 0 & 0 \\
\hline & 0 & 3 & 6 & 0 & 0 & 0 & 0 & 3 & 6 & 0 & 0 & 0 & 0 \\
\hline & 0 & 5 & 6 & 0 & 0 & 0 & 5 & 0 & 4 & 0 & 0 & 0 & 4 \\
\hline & 6 & 7 & 10 & 0 & 10 & 0 & 0 & 2 & 7 & 0 & 0 & 0 & 2 \\
\hline & 0 & 0 & 9 & 0 & 0 & 0 & 0 & 0 & 0 & 0 & 0 & 0 & 0 \\
\hline Eté & 0 & 6 & 8 & 0 & 0 & 2 & 0 & 0 & 0 & 0 & 0 & 0 & 0 \\
\hline & 0 & 7 & 11 & 0 & 0 & 2 & 0 & 0 & 2 & 0 & 0 & 5 & 5 \\
\hline & 0 & 6 & 9 & 2 & 3 & 0 & 4 & 0 & 0 & 0 & 0 & 2 & 7 \\
\hline & 4 & 5 & 8 & 0 & 9 & 6 & 0 & 5 & 9 & 0 & 7 & 0 & 0 \\
\hline & 0 & 0 & 1 & 0 & 0 & 0 & 0 & 0 & 0 & 0 & 0 & 0 & 0 \\
\hline Automne & 0 & 9 & 10 & 0 & 0 & 0 & 0 & 0 & 4 & 0 & 3 & 0 & 0 \\
\hline & 0 & 10 & 13 & 0 & 0 & 3 & 0 & 5 & 5 & 1 & 4 & 2 & 4 \\
\hline & 2 & 10 & 12 & 0 & 4 & 0 & 8 & 4 & 4 & 2 & 5 & 1 & 6 \\
\hline & 3 & 6 & 7 & 0 & 6 & 7 & 0 & 4 & 8 & 0 & 4 & 0 & 0 \\
\hline & 0 & 3 & 6 & 0 & 0 & 5 & 0 & 4 & 3 & 0 & 1 & 0 & 0 \\
\hline Hiver & 0 & 0 & 3 & 0 & 0 & 1 & 0 & 1 & 0 & 0 & 0 & 0 & 0 \\
\hline & 0 & 6 & 10 & 0 & 0 & 5 & 1 & 3 & 5 & 0 & 2 & 0 & 0 \\
\hline & 1 & 9 & 11 & 0 & 3 & 6 & 8 & 3 & 5 & 2 & 5 & 0 & 0 \\
\hline
\end{tabular}

Les données physico-chimiques (Tableau II) permettent de rendre compte du niveau de pollution organique à chaque station. La station 1 est la station témoin théoriquement exempte de pollution organique. La station 2 est marquée par de fortes valeurs de DBO5 et de concentrations en ammoniaque, nitrates et phosphates et une nette diminution de la concentration en oxygène. Cette station est soumise à l'impact des effluents domestiques du village d'Autrans (Figure 2A). La station 3 située $3,3 \mathrm{~km}$ plus en aval présente encore 
des valeurs fortes de DBO5 ; l'apparition de plus fortes concentrations en nitrates souligne la mise en place du processus de nitrification. Ce processus se poursuit plus en aval (stations 4 et 5 situées respectivement à $5,7 \mathrm{~km}$ et $9,7 \mathrm{~km}$ de la station 2). La valeur élevée de DBO5 à la station 4 est un mélange des effets des effluents des villages d'Autrans et de Méaudre. Au vu des résultats, ce dernier rejet a cependant un impact négligeable au niveau de la station 4.

\section{Tableau II}

Valeurs moyennes (moy) et écart types (Et) de 10 paramètres physico-chimiques mesurés à quatre reprises sur les 5 stations du Méaudret (d'après PEGAZ-MAUCET, 1980).

\section{Table II}

Means (moy) and standard deviations (Et) of 10 physical and chemical parameters measured at four dates in the five sites of the Meaudret (from PEGAZ-MAUCET, 1980).

\begin{tabular}{|c|c|c|c|c|c|c|c|c|c|c|}
\hline \multirow[t]{2}{*}{ Stations } & \multicolumn{2}{|l|}{1} & \multicolumn{2}{|c|}{2} & \multicolumn{2}{|c|}{3} & \multicolumn{2}{|c|}{4} & \multicolumn{2}{|c|}{5} \\
\hline & moy & Et & moy & Et & moy & Et & moy & $\mathrm{Et}$ & moy & Et \\
\hline Température $\left({ }^{\circ} \mathrm{C}\right)$ & 6.8 & 5.7 & 7.5 & 5.3 & 7.8 & 6.3 & 8.5 & 6.6 & 8.0 & $\overline{7.0}$ \\
\hline Débit $(1 / s)$ & 61.5 & 40.6 & 138.3 & 86.4 & 173.0 & 107.9 & 250.8 & 184.1 & 236.0 & 145.8 \\
\hline $\mathrm{pH}$ & 8.3 & 0.2 & 8.1 & 0.3 & 8.2 & 0.3 & 8.3 & 0.2 & 8.4 & 0.2 \\
\hline Conductivité $(\mu \mathrm{S} / \mathrm{cm})$ & 315 & 14 & 370 & 46 & 349 & 42 & 328 & 29 & 316 & 27 \\
\hline Oxygène (mg/l) & 9.9 & 8.2 & 4.3 & 39.6 & 8.6 & 29.0 & 10.2 & 20.6 & 10.0 & 12.3 \\
\hline DBO5 (mg/l d'O2) & 2.0 & 0.4 & 18.5 & 13.1 & 8.5 & 4.9 & 5.6 & 4.4 & 2.2 & 1.0 \\
\hline Oxydabilité (mg/l d'O2) & 1.2 & 0.5 & 5.0 & 2.4 & 2.2 & 0.6 & 1.8 & 0.6 & 1.4 & 0.4 \\
\hline Ammoniaque (mg/l) & 0.12 & 0.04 & 6.92 & 5.01 & 3.45 & 3.17 & 1.70 & 2.15 & 0.34 & 0.22 \\
\hline Nitrates $(\mathrm{mg} / \mathrm{l})$ & 3.65 & 1.96 & 2.58 & 1.57 & 7.43 & 4.17 & 7.20 & 3.77 & 7.28 & 2.87 \\
\hline Phosphates (mg/l) & 0.12 & 0.07 & 3.31 & 2.34 & 2.79 & 1.91 & 1.65 & 1.26 & 1.11 & 0.50 \\
\hline
\end{tabular}

\section{RÉSULTATS}

\section{Analyse triadique partielle sur $K$ tableaux faunistiques centrés par espèce}

On considère d'abord les tableaux faunistiques comme représentant $K$ tableaux d'ACP à analyser simultanément. Sur l'exemple traité ici (Tableau I), on effectue donc initialement un centrage des données par colonne (la normalisation est ici inutile du fait de la nature des données étudiées). La représentation graphique de la distribution spatiotemporelle des taxons permet de visualiser des tendances qu'il faut expliciter et associer par l'analyse (Figure 2).

Interstructure et recherche du compromis. La matrice des corrélations vectorielles entre tableaux-dates (Tableau III) montre que la structure du tableau correspondant au printemps est la plus éloignée des trois autres. La meilleure ressemblance est observée entre les tableaux été et automne pour lesquels la valeur du coefficient Rv est la plus élevée $(0,687)$. Dans le Tableau III on trouve également : (1) les coefficients $\alpha \cdot k$, qui représentent le poids du tableau $k$ dans la définition du compromis, sont calculés de façon à ce que le tableau compromis ait une inertie maximale, (2) la variance vectorielle du tableau $k$ définit le degré de structure propre à chaque tableau et (3) le $\operatorname{Cos}^{2}$ entre le tableau $k$ et le compromis qui constitue un indice de la qualité de l'expression par une date donnée de la structure du compromis. Ces indices typologiques montrent d'une part que le tableau automne est celui qui participe le plus à la définition du compromis, qu'il a la plus forte structure et qu'il est le mieux représenté par le compromis (valeurs élevées de $\alpha_{k}$, de 
variance vectorielle et de $\operatorname{Cos}^{2}$ ) et d'autre part que le tableau printemps participe le moins à la construction du compromis car il présente la structure la plus faible et il est de fait le moins bien représenté.
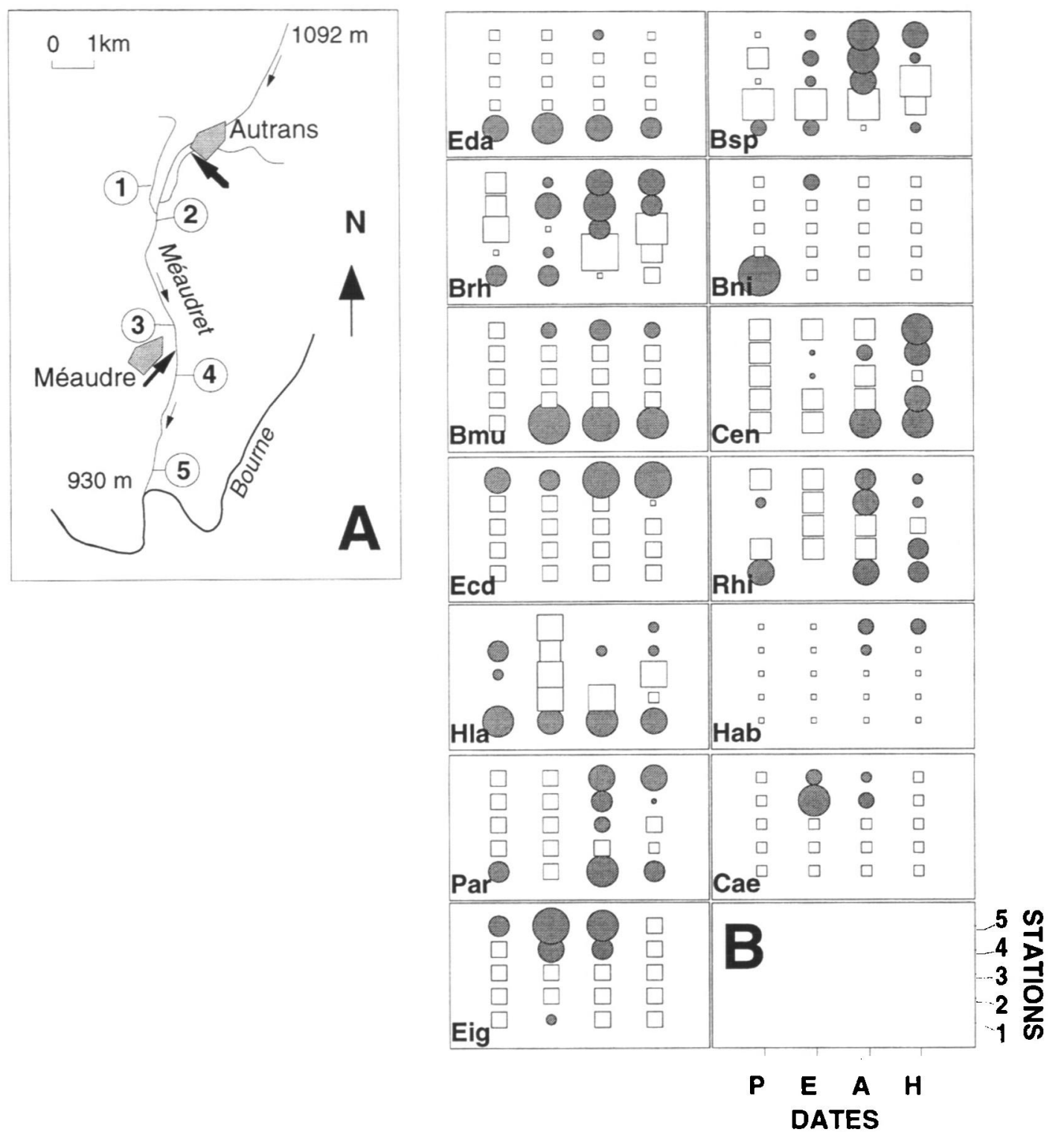

\section{Figure 2}

(A) Stations d'échantillonnages. Les zones grisées (villages) constituent les principales sources de pollution organique (taille des flèches proportionnelle au rejet). (B) Représentation spatio-temporelle des abondances des 13 taxons d'Ephéméroptères. La surface des cercles (valeurs supérieures à la moyenne) et des carrés (valeurs inférieures à la moyenne) est proportionnelle aux données centrées (les codes des taxons sont dans le Tableau I).

Figure 2

(A) Study sites. Dotted areas (villages) represent the main sources of organic pollution (arrow size proportional to the effluent). (B) Spatio-temporal representation of the abundance of the 13 Ephemeropteran taxa. The surface of circles (values $>$ mean) and squares (values $<$ mean) is proportional to the centred values (see Table I for taxa codes). 


\section{Tableau III}

Paramètres numériques associés à l'analyse triadique partielle des données faunistiques. $\mathrm{RV}=$ matrice des coefficients de corrélations vectorielles entre tableaux $; \alpha_{k}=$ pondération indiquant la participation du tableau $k$ à la définition du compromis ; Var. vect. = variance vectorielle mesurant l'inertie de chaque tableau ; $\operatorname{Cos}^{2}$ : cosinus carré entre le tableau $k$ et le compromis réduit à sa partie interprétée, ici suivant deux axes.

\section{Table III}

Numerical parameters associated with partial triadic analysis processed on the faunistic data. RV = correlation matrix between tables; $\alpha_{k}=$ weights of tables in the definition of the compromise Var. vect. = norm-squared or inertia of each table $; \operatorname{Cos}^{2}=$ square cosine between a table and the approximated compromise using two axes.

\begin{tabular}{|c|c|c|c|c|c|c|c|}
\hline Tableau & \multicolumn{4}{|c|}{ RV } & $\alpha_{k}$ & Var. & $\operatorname{Cos}^{2}$ \\
\hline Printemps & 1 & & & & 0,278 & 3,114 & 0,267 \\
\hline Eté & 0,468 & 1 & & & 0,484 & 4,043 & 0,579 \\
\hline Automne & 0,368 & 0,687 & 1 & & 0,709 & 6,997 & 0,705 \\
\hline Hiver & 0,320 & 0,499 & 0,612 & 1 & 0,432 & 3,914 & 0,583 \\
\hline
\end{tabular}
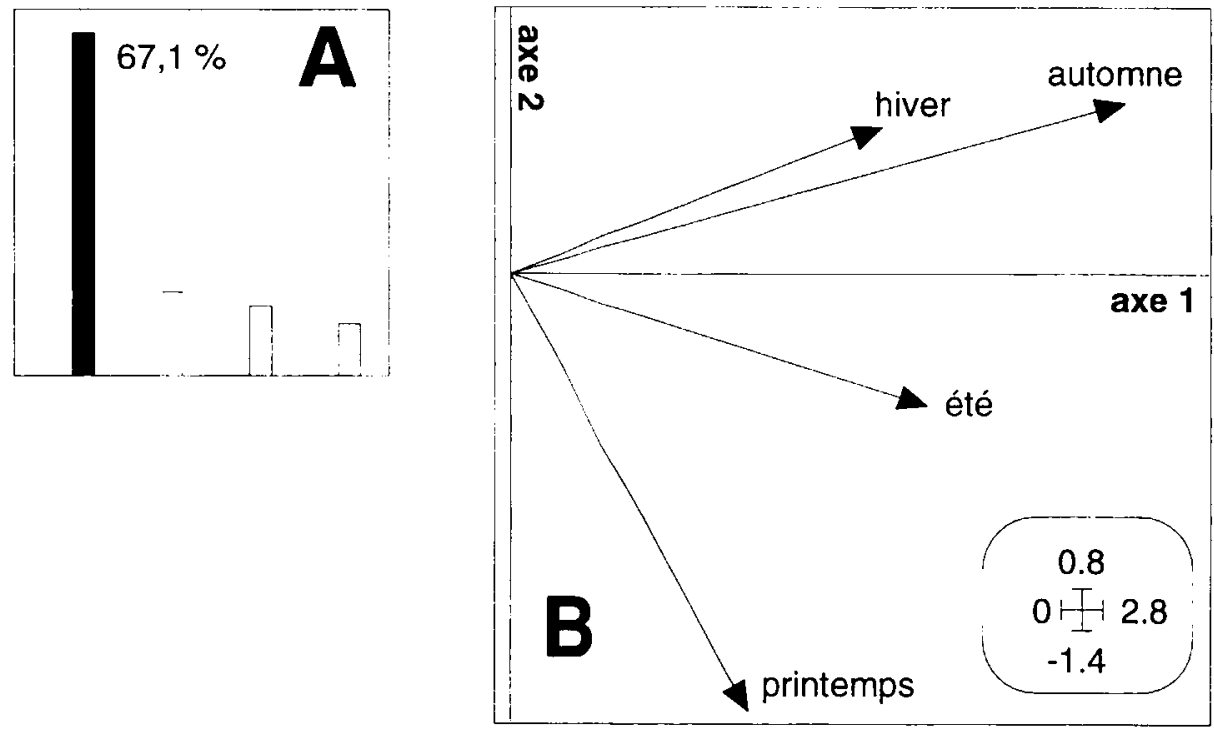

Figure 3

Interstructure de l'analyse triadique partielle des données faunistiques centrées par taxon. (A) Histogramme des valeurs propres issues de la diagonalisation de la matrice des covariances vectorielles. (B) Projections des quatre tableaux (saisons) sur les axes 1 et 2 de l'interstructure.

Figure 3

Interstructure results of the partial triadic analysis processed on the faunistic data centred by taxon. (A) Histogram of eigenvalues generated by the diagonalization of the matrix that contains table covariance. (B) Projections of the four tables (seasons) on the interstructure axes 1 and 2. 
La diagonalisation de la matrice de covariances vectorielles (Covv) fournit des axes principaux (Figure 3A) dont le premier représente $67,1 \%$ de l'inertie. La représentation des vecteurs propres (à un coefficient $\sqrt{\lambda_{k}}$ près) de la matrice des Covv fournit une image euclidienne de la ressemblance entre tableaux mesurée par les Covv. A l'examen des projections sur le premier axe qui a une fonction de définition d'une moyenne, on retrouve les résultats donnés par les valeurs des $\alpha_{k}$ (Tableau III) : l'automne se distingue sensiblement des autres dates par sa coordonnée la plus élevée sur l'axe 1 d'interstructure et le printemps par sa coordonnée la plus faible (Figure 3B). A ce stade, le tableau compromis, somme pondérée par les coefficients $\alpha_{k}$, des tableaux-dates, est donc constitué

Analyse du compromis. L'analyse du tableau compromis fournit un histogramme de valeurs propres (Figure 4A) soulignant deux dimensions prépondérantes. Fondamentalement, les poids des tableaux sont choisis pour que la somme des valeurs propres du compromis soit optimale, c'est-à-dire pour que le tableau compromis ait une inertie maximale. Taxons d'Ephéméroptères (Figure 4B) et stations (Figure 4C) sont donc projetés sur le plan 1-2 du compromis.

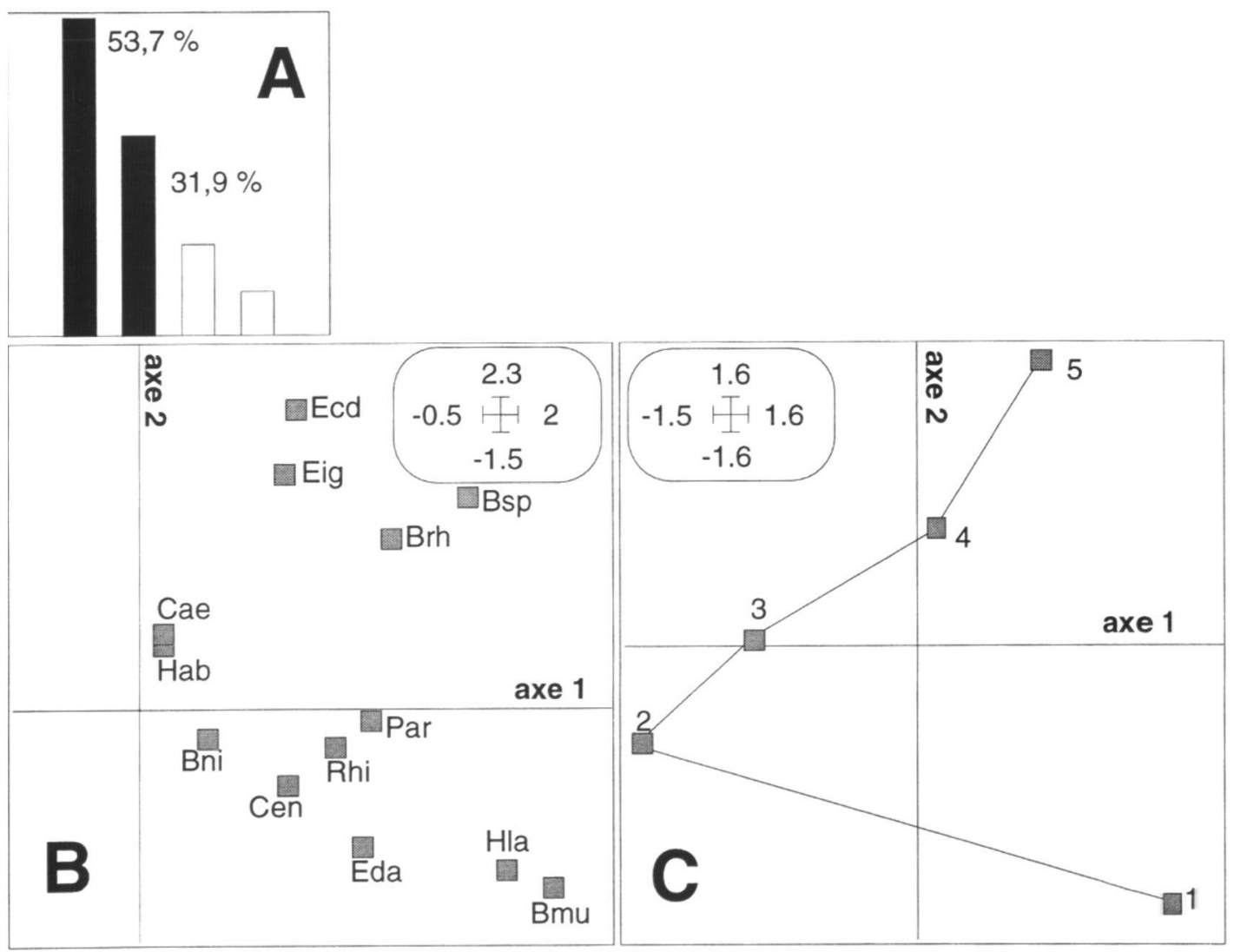

Figure 4

Résultats de l'analyse du compromis. (A) Histogramme des valeurs propres soulignant l'existence d'une structure moyenne à deux dimensions. (B) Coordonnées des taxons d'Ephéméroptères sur le plan 1-2 du compromis (les codes des espèces sont dans le Tableau I). (C) Coordonnées des stations sur le plan 1-2 du compromis.

\section{Figure 4}

Results of the compromise analysis. (A) Histogram of eigenvalues identifying the prominence of the two first axes that define the average structure. (B) Coordinates of the Ephemeropteran taxa on the first compromise plane (see Table I for taxa codes). (C) Coordinates of the stations on the first compromise plane. 
Le plan 1-2 des stations (Figure 4C) met en évidence sur l'axe 1 un gradient de pollution opposant la station 1 (témoin) et les stations 4 et 5 éloignées des rejets organiques du village d'Autrans (Figure 2A) aux stations 2 et 3 subissant l'impact du rejet. L'axe 2 caractérise le gradient amont-aval.

Le long de l'axe 1, la carte des espèces (Figure 4B) est caractéristique d'un effet taille (taxons situés du côté positif de l'axe 1), c'est-à-dire que tous les taxons sont abondants ou rares en même temps. Ceci peut s'expliquer par le fait que les stations 2 et 3 (situées du côté négatif de l'axe 1, Figure 4C) sont globalement pauvres en Ephéméroptères par rapport aux autres stations. On peut confirmer ce résultat en calculant un indice d'abondance moyenne en Ephéméroptères par station. On obtient respectivement $3,7,2,5$ et 3,2 pour les stations 1,4 et $5,0,8$ et 1,2 pour les stations 2 et 3 .

Le long de l'axe 2, qui représente $31,9 \%$ de l'information totale, les taxons s'ordonnent suivant un gradient longitudinal. En se référant également à la représentation de la répartition des espèces (Figure 2), on peut interpréter le compromis de la façon suivante : les taxons E. danica (Eda), Baetis muticus (Bmu) et Habrophlebia lauta (Hla) se trouvent majoritairement (et relativement puisque les données ont été initialement centrées) dans la station 1 témoin la plus en amont alors que les taxons Ephemerella ignita (Eig) et d'Ecdyonurus sp. (Ecd) caractérisent les stations 4 et 5 les plus en aval où le cours d'eau amorce sa restauration. Ces cinq espèces présentent, d'une part des exigences quant aux conditions d'habitat (amont-aval), d'autre part une certaine sensibilité à la pollution organique. Les taxons Baetis sp. (Bsp) et Baetis rhodani (Brh) sont trouvés en abondance à la fois en aval (stations 4 et 5) mais également dans la station témoin, ce qui explique leur position. Les taxons Habroleptoides sp. (Hab) et Caenis sp. (Cae) présents uniquement dans les stations 4 et 5 pourraient caractériser ces dernières mais leur abondance générale très faible est à l'origine de leur position sur le plan. Enfin, les taxons Paraleptophlebia sp. (Par), Rhithrogena sp. (Rhi) et Centroptilum sp. (Cen) sont moyennement abondants; on les trouve cependant plus fréquemment en amont qu'en aval.

Analyse de la reproductibilité de la structure du compromis. La reproductibilité de la structure compromis dans le temps peut être représentée pour les taxons (Figure 5A) et les stations (Figure $5 \mathrm{C}$ ) et va permettre de discuter de l'évolution temporelle des relations taxons-stations (typologie interne à chaque tableau). Pour cela, on projette sur le plan 1-2 du compromis analysé ci-dessus les lignes (Figure 5C), les colonnes (Figure 5A), les axes (Figure 5D) et les composantes principales (Figure 5B) issus des analyses séparées de chacun des tableaux.

Au printemps, les peuplements appauvris des stations 3 et 4 sont proches de la station 2. Notons en effet l'absence totale de Centroptilum sp. (Cen), Habroleptoides $s p$. $(\mathrm{Hab})$, Baetis muticus (Bmu) et de Caenis sp. (Cae) (concentrées au centre du plan) et la diminution de Baetis rhodani (Brh) et de Baetis sp. (Bsp). II semble donc qu'en cette saison, il y ait une diminution nette de l'abondance générale d'Ephéméroptères liée aux phases d'émergence. En été, la station 4 retrouve Caenis $s p$. (Cae) et Centroptilum sp. (Cen) absents en juin. De plus, le peuplement caractéristique de la station 1 ( $E$. danica ( $E d a)$, Baetis muticus (Bmu) et Habrophlebia lauta (Hla)) se distingue à nouveau nettement. En automne, la situation est quasiment la même que celle du compromis. Seul Baetis niger (Bni) est absent et on retrouve des taxons caractéristiques des stations 4 et 5 Ephemerella ignita (Eig) et Ecdyonorus sp. (Ecd). En hiver, la situation se dégrade à nouveau, en particulier pour les stations 3 et 4. Des taxons tels que Ephemerella ignita (Eig), Caenis sp. (Cae) et Baetis niger (Bni) ont totalement disparu et globalement toutes les autres espèces voient leur abondance diminuer, ce qui se caractérise par une évolution de la position de toutes les stations du côté négatif de l'axe 1. II est possible que les forts débits de février décalent davantage la pollution vers les stations situées en aval, ce qui expliquerait que la pollution affecte plus la station 3 que la 2 (PEGAZ-MAUCET, 1980). 


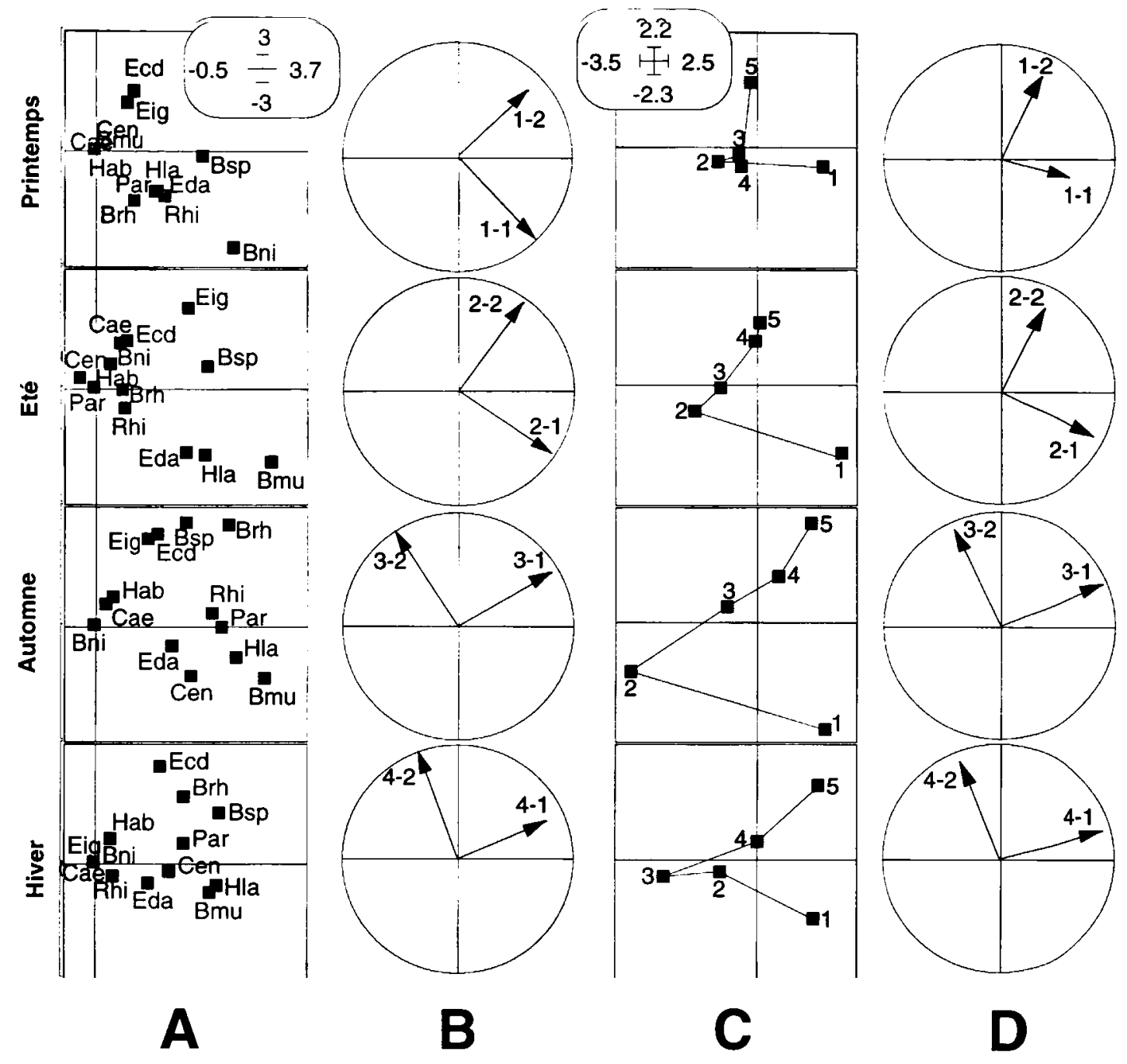

Figure 5

Analyse de la reproductibilité de la structure compromis. (A) Projection multifenêtrée par tableau (dates) des colonnes (taxons) sur le plan 1-2 du compromis (Cf. Tableau I pour les codes des taxons). (B) Projection multifenêtrée par tableau des composantes principales des analyses séparées sur le plan 1-2 du compromis. Le premier caractère identifie le numéro du tableau, le second représente le numéro de l'axe de l'analyse séparée correspondante. (C) Projection multifenêtrée par tableau des lignes (stations) sur le plan 1-2 du compromis. (D) Projection multifenêtrée par tableau des axes principaux des analyses séparées sur le plan 1-2 du compromis. Le premier caractère identifie le numéro du tableau, le second représente le numéro de l'axe de l'analyse séparée.

\section{Figure 5}

Analysis of the reproductibility of the compromise structure. (A) Multiwindowed projection (by date) of columns (taxa) on the first compromise plane (see Table I for taxa codes). (B) Multiwindowed projection (by table) of the first four principal components of the separate analyses on the first compromise plane. The first character identifies the table number and the second represents the axis number of the corresponding separate analysis. (C) Multiwindowed projection (by date) of rows (stations) on the first compromise plane. (D) Multiwindowed projection (by date) of the first four principal axes of the separate analyses on the first compromise plane. 
Les projections des composantes principales (Figure 5B) et des axes principaux (Figure 5D) des analyses réalisées séparément par date sur le plan 1-2 du compromis permettent de visualiser la contribution de chacun des tableaux-dates à la formation du compromis. En effet, par simple rotation on peut superposer les axes 1-2 des analyses séparées aux axes 1-2 du compromis et plus les extrémités des flèches sont proches du cercle de rayon unité, meilleure est cette contribution. Ces projections sont très semblables et confirment les résultats issus de l'analyse de l'interstructure. Ainsi, on y retrouve le fait principal que l'analyse est centrée autour du tableau automne qui est le mieux représenté (par rotation les composantes principales (Figure 5B) et les axes principaux (Figure 5D) se superposent aux axes du compromis et les extrémités des flèches sont très proches du cercle de rayon unité).

\section{AFC moyenne de FOUCART}

Sur le même cortège faunistique, on peut éliminer les effets parasites dûs aux variations d'abondance liées à la pollution. En choisissant une stratégie d'AFC sur le même jeu de données, on exprime explicitement que l'on veut faire une typologie des courbes de réponse des invertébrés au gradient longitudinal et que l'on recherche également une évolution temporelle de cette typologie. L'AFC du tableau compromis en œuvre ici peut être interprétée de la même manière qu'une AFC classique. On peut reprendre alors les propriétés d'ordination sur gradient de cette méthode largement explicitées par THIOULOUSE et CHESSEL (1992). Dans une AFC classique, les premiers scores factoriels des lignes et des colonnes présentent une corrélation maximale (WILLIAMS, 1952 ; HILL, 1973). De façon similaire, les scores factoriels des lignes (stations) et des colonnes (taxons) résultant de l'AFC de FOUCART peuvent être utilisés pour réordonner le tableau compromis (Figure 6). Les distributions stationnelles des espèces sont résumées par le calcul d'une moyenne conditionnelle (cercles noirs sur la Figure 6 ) assortie de \pm un ècart type (segments sur la Figure 6) autour de celle-ci. Dans notre exemple, on obtient une image synthétique des distributions longitudinales ordonnées des taxons.

La projection des relevés en individus supplémentaires sur le plan du compromis (Figure 7A) souligne une certaine convergence de structure longitudinale d'une date à une autre. La station 1 est séparée en permanence de la station 5 tandis que les stations 2, 3 et 4 s'ordonnent de l'amont vers l'aval avec plus ou moins de chevauchement (Figure 7B). La projection des taxons en individus supplémentaires reproduit la structure compromis avec trois groupes de taxons plus ou moins nettement séparés à chaque date (Figure $7 \mathrm{C}$ ).

On peut noter par exemple un glissement de la position de Baetis niger de l'amont vers l'aval entre le printemps et l'été ou de la position de Paraleptophlebia sp. du printemps à l'hiver. Des taxons présentent des profils plus stables tels que Ephemera danica ou Baetis muticus à l'amont ou Ecdyonurus sp. à l'aval. II est remarquable de noter qu'en dépit de ces modifications faunistiques la structure organisée autour du gradient longitudinal et de l'impact du rejet polluant (Figure 7A) est relativement stable.

\section{CONCLUSION}

A travers l'illustration choisie nous avons montré que l'analyse triadique partielle, qui autorise la mise en œuvre de plusieurs ACP simultanées, permet de mettre en évidence par l'analyse d'un compromis une structure spatiale moyenne par exemple. L'étape suivante de l'analyse de la reproductibilité de cette structure moyenne par chacun des $K$ tableaux permet d'étudier son évolution dans le temps. On montre ainsi sur notre exemple que l'étude du compromis met en évidence une typologie espèces-stations de référence caractérisée d'une part par une pauvreté faunistique des stations soumises à la pollution et d'autre part par un contenu taxonomique évoluant longitudinalement. L'étude de la reproductibilité de la 


\section{TAXONS}

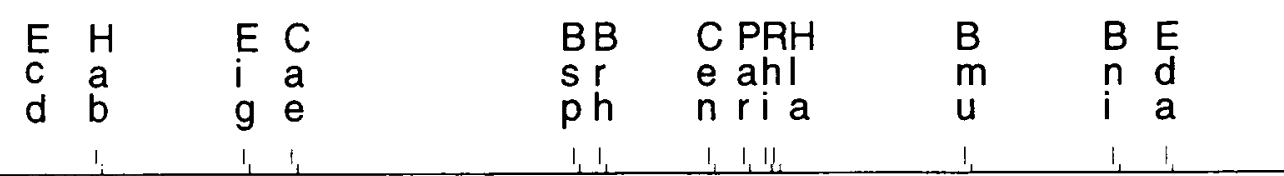

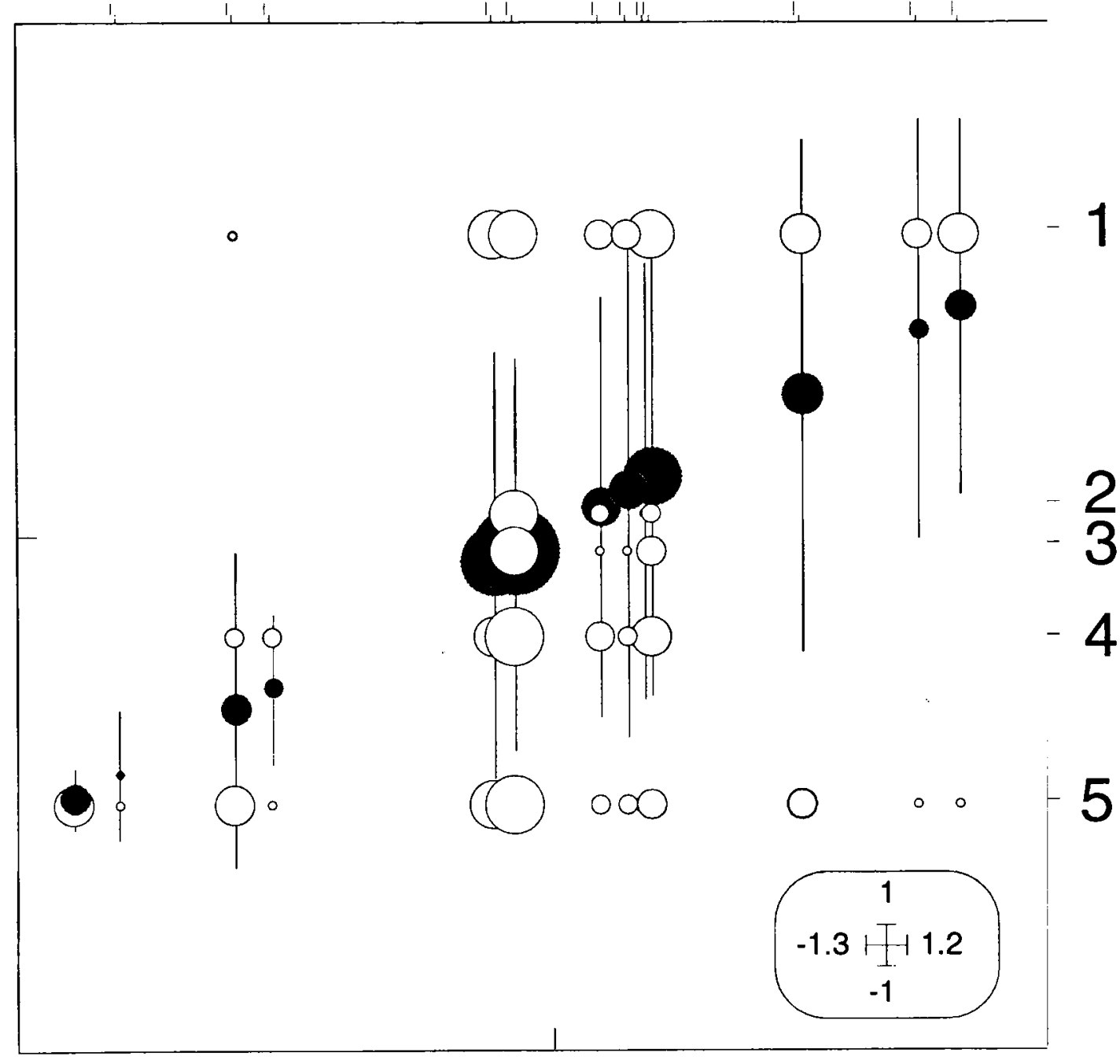

Figure 6

Représentation graphique du tableau compromis stations-taxons après une AFC de FOUCART. Les stations (en ordonnées) et les taxons (en abscisses) sont ordonnés suivant leur premier score factoriel. La taille des cercles en blanc est proportionnelle à l'abondance du taxon dans un site. On a superposé à cette image les positions moyennes pondérées des taxons (cercles noirs) sur le premier score factoriel des stations. Les segments de part et d'autre de cette moyenne représentent un écart type autour de la moyenne.

Figure 6

Graphical representation of the taxa-by-stations compromise table after a FOUCART's correspondence analysis. Stations (ordinates) and taxa (abscissas) were ordered along the first axis score of the compromise analysis. White circles are proportional to the abundance of taxa. Weighted average positions of taxa were added (black circles) using the first station scores of the analysis. Lines represent one standard deviation around the mean. 

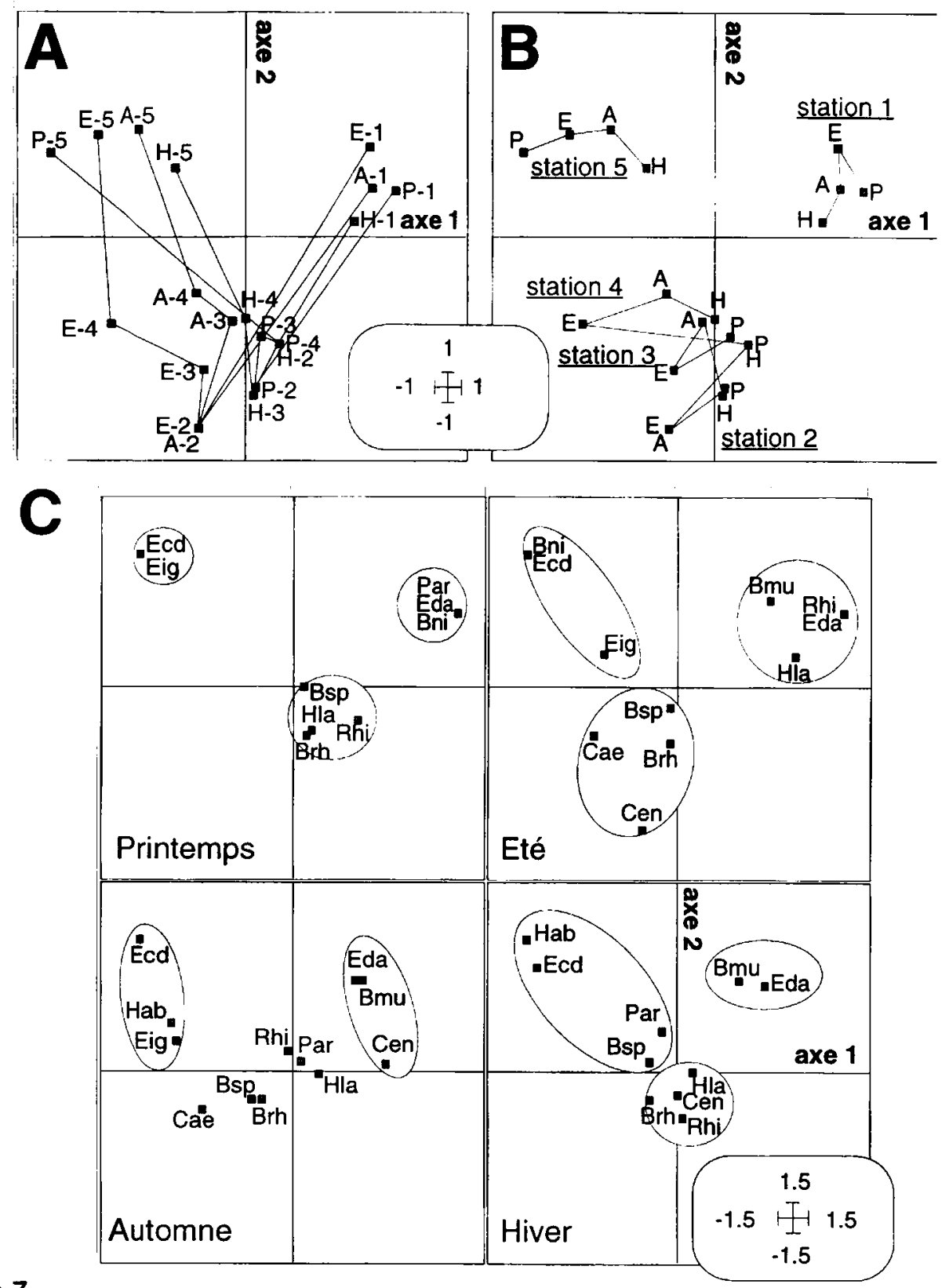

Figure 7

(A) Projections des relevés sur le plan 1-2 de la structure compromis. Une étiquette comporte en premier l'initiale de la saison et en second le numéro de la station. Les trajectoires correspondent aux 4 dates. (B) Même représentation mais les trajectoires correspondent ici aux 5 stations. (C) Projections des taxons par leur position moyenne (à une constante près) de chaque tableau. Les points à l'origine correspondant aux taxons absents à une date donnée ont été éliminés.

\section{Figure 7}

(A) Projections of samples on the first factorial plane of the compromise table. The character strings incorporate a letter for identifying the season and a number for identifying the station. Trajectories correspond to the 4 dates. (B) Same graphical presentation except that trajectories correspond to the 5 stations. (C) Projections of the taxa at their weighted average (at about a coefficient) in each table. Points at the origin corresponding to taxa that were absent at a given date were removed from the graph. 
structure moyenne observée permet de décrire l'évolution saisonnière de la typologie stationnelle et du cortège faunistique.

D'un point de vue écologique, cette méthode d'analyse multitableaux autorise donc une véritable appréhension de la dynamique d'un peuplement faunistique ou une réelle approche fonctionnelle d'un système. THIOULOUSE et CHESSEL (1987) avaient alors présenté la méthode en approfondissant l'analyse de l'interstructure, étape précédant celle de l'analyse du compromis. Or, l'objectif principal de la méthode est la recherche et l'analyse d'une structure moyenne. C'est pourquoi on ne retient plus ici de l'interstructure que le premier vecteur propre qui a seul une définition de moyenne alors que les suivants caractérisent l'écart au modèle moyen et nous considérons donc qu'il n'y a pas lieu de les prendre en compte. Ce choix devrait simplifier l'usage de la méthode pour les utilisateurs.

Dans les mêmes conditions expérimentales que celles qui président à l'analyse triadique partielle, on peut choisir de ne pas prendre en compte les variations d'abondance des taxons et donc implicitement d'éliminer les effets de la pollution. Ce choix revient à opter pour une stratégie d'AFC. L'AFC moyenne de FOUCART permet de comparer plusieurs tableaux de contingence. Cette méthode a l'avantage de résoudre le problème des pondérations lié à ce type d'analyse et ne présente pas de difficultés techniques majeures pour l'utilisateur. Par contre, dans l'étude des cubes de données construits à partir de données stations-dates-variables le choix du point de vue dépend des objectifs expérimentaux de l'utilisateur et peut mener à des résultats très différents. En effet, six stratégies d'analyses sont possibles en fonction de l'organisation initiale des tableaux (Figure 8). Du fait de la symétrie, l'usage de l'AFC conduit à trois stratégies dont les compromis sont respectivement du type espèces-stations, espèces-dates et stations-dates.

L'analyse triadique partielle impose que chaque tableau pris en compte ait la même dimension (même nombres de lignes et de colonnes) c'est-à-dire que les mesures portent sur les mêmes descripteurs et les mêmes individus à chaque réplicat. Si ce n'est pas le cas, on aura recours à d'autres analyses multitableaux comme l'ACT-STATIS sur les opérateurs (LAVIT et al., 1994), l'analyse factorielle multiple (ESCOFIER et PAGĖS, 1994) ou l'analyse de co-inertie multiple (CHESSEL et HANAFI, 1996) dont les fondements théoriques sont en revanche plus complexes.

Dans la continuité de ce travail et pour répondre aux questions des influences des échelles spatiales et temporelles sur les cortèges floro-faunistiques, il conviendra de développer l'utilisation des analyses multitableaux dans le cadre des relations fauneenvironnement. La caractérisation des relations entre un tableau faunistique et un tableau mésologique est en effet un problème central de l'écologie des communautés (CHESSEL et MERCIER, 1993). L'analyse de co-inertie (DOLÉDEC et CHESSEL, 1994) est une méthode de couplages de tableaux utilisable pour tous les types de données qui permet d'étudier la co-structure entre des relevés faunistiques et des relevés mésologiques. L'objectif de cette analyse est de trouver ce qui, dans deux groupes de descripteurs, engendre une typologie commune des objets décrits dans chacun des tableaux. A ce titre, la méthode STATICO (CHESSEL et al., 1996 ; SIMIER et al., 1999), en combinant la logique de l'analyse triadique partielle et la logique des couplages de co-inertie, permet de traiter simultanément une série de $K$ couples de tableaux. Cette possibilité ouvre des perspectives intéressantes pour l'étude de la stabilité des relations entre faune et environnement.

La diffusion de ces méthodes sera poursuivie dans le cadre du Réseau Hydrobiologique et Piscicole (RHP), mis en place à l'échelle nationale par le Conseil Supérieur de la Pêche. En effet, celui-ci constitue un réseau de suivi piscicole réalisé au travers d'un ensemble de stations échantillonnées depuis 4 ou 5 ans. L'analyse triadique partielle et l'AFC de FOUCART seront utilisées en particulier afin de voir si l'on peut définir une structure stationnelle " moyenne " et comment cette structure évolue temporeliement (BLANC et BEAUDOU, 1998). 
<smiles>O=S</smiles>

Espèces
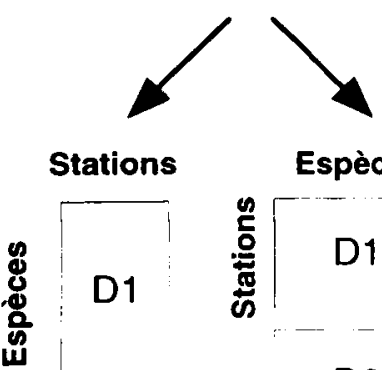

$\mathrm{D2}$

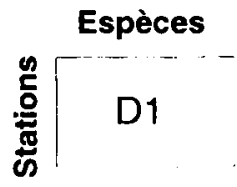

$\mathrm{D} 2$

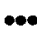

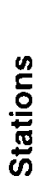

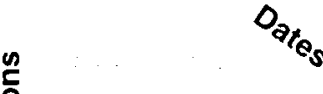

Espèces
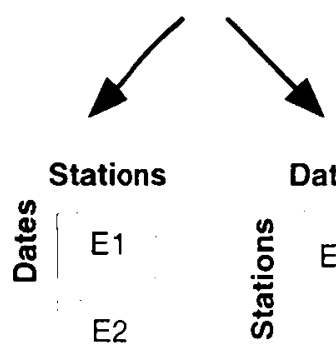

$\cdots$

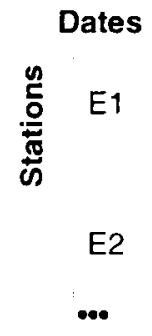

Dates

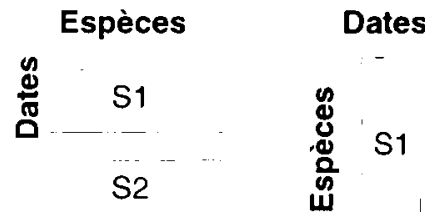

S2

\section{Figure 8}

Les six dispositions fondamentales associées à un tableau faunistique ternaire. L'usage de l'AFC induit trois analyses alternatives de points de vue différents. Dans cet article, nous n'avons abordé que le premier cas ( $D$ pour date, $E$ pour espèce, et $S$ pour station).

\section{Figure 8}

The six arrangements associated to a ternary faunistic matrix. The use of correspondence analysis induces three approaches linked to a particular point of view. In this paper, we have analysed the first arrangement only ( $D$ for date, $E$ for species, and $\mathbf{S}$ for station).

Les aides à l'interprétation proposées dans cet article sont issues du logiciel ADE-4 (THIOULOUSE et al., 1997) librement diffusé sur Internet (http://pbil.univ-lyon1.fr/ADE-4/ ADE-4F.html). Les représentations multifenêtrées par tableau sont tout à fait dans la logique des analyses multitableaux puisqu'elles permettent de visualiser de façon coordonnée les lignes et les colonnes de chacun des tableaux dans le plan compromis. Ces fonctions graphiques multifenêtrées ainsi que les calculs des analyses multitableaux citées ci-dessus peuvent être mis en œuvre à l'aide de la programmathèque ADE-4.

\section{REMERCIEMENTS}

Ce travail a été réalisé dans le cadre d'une convention d'étude financée par le Conseil Supérieur de la Pêche (CSP) et établie entre le C.N.R.S., le Laboratoire d'Hydrobiologie Marine et Continentale de l'Université Montpellier II dirigé par Monsieur le Professeur G. LASSERRE et le CSP. Nous remercions Messieurs E. VIGNEUX, Chef du Service de l'Informatique et des Relations Scientifiques du CSP et G. LASSERRE sans lesquels l'élaboration de cette convention n'aurait pas eu lieu ainsi que les membres de la Délégation Régionale $n^{\circ} 8$ du CSP et en particulier Monsieur J. MIELOT, délégué régional, et D. BEAUDOU, chargé d'études, pour leur accueil dans leurs locaux et leur soutien actif à ce travail et enfin M. SIMIER, ingénieur à l'ORSTOM, pour son aide et ses conseils. 


\section{BIBLIOGRAPHIE}

ALIAUME C., MONTEIRO C., LOUIS M., LAM HOAI T., LASSERRE G, 1993. Organisation spatio-temporelle des peuplements ichtyologiques dans deux lagunes côtières : au Portugal et en Guadeloupe. Acta CEcologica, CEcologia Generalis, 16, 3, 291-301.

AMANIEU M., GUELORGET O., NOUGIER-SOULE J., 1981. Analyse de la diversité de la macrofaune benthique d'une lagune littorale méditerranéenne. Vie Milieu, 31, 303-312.

AMBLARD C., CARRIAS J.F., BOURDIER G., MAURIN N., 1995. The microbial loop in a humid lake : seasonal and vertical variations in the structure of the different communities. Hydrobiologia, 300/301, 71-84.

BAGLINIĖRE J.L., MAISSE G., LEBAIL P.Y., PRÉVOST E., 1987. Dynamique de la population de la truite commune Salmo trutta d'un ruisseau breton, France. Acta CEcologica, CEcologia Generalis, 8, 3, 201-215.

BARAN E., 1995. Dynamique spatio-temporelle des peuplements de poissons estuariens en Guinée, Afrique de l'Ouest. Thèse de Doctorat, Université de Bretagne Occidentale, $204 \mathrm{p}$.

BEFFY J.L., DOLÉDEC S., 1991. Mise en évidence d'une typologie spatiale dans le cas d'un fort effet temporel : un exemple en hydrobiologie. Bulletin d'Ecologie, 22, 3-11.

BENGEN D., LIM P., BELAUD A., 1992. Qualité des eaux de trois bras morts de la Garonne : variabilité spatio-temporelle. Revue des Sciences de l'Eau, 5, 131-156.

BIRKS H.J.B., PEGLAR S.M., AUSTIN A.A., 1994. An annotated bibliography of canonical correspondence analysis and related constrained ordination methods 1986-1993. Botanical Institute, Université de Bergen, $58 \mathrm{p}$.

BLANC L., BEAUDOU D., 1998. Stabilité temporelle des structures spatiales des peuplements piscicoles des régions Languedoc-Roussillon et Provence-Alpes-Côte d'Azur. Bull. Fr. Pêche Piscic., 348.

BOET P., ALLARDI J., LEROY J., 1991. Le peuplement ichtyologique du bassin de I'Yonne. Bull. Fr. Pêche Piscic., 320, 7-28.

BORNETTE G., AMOROS C., COLLILIEUX G., 1994. Role of seepage supply on the aquatic vegetation dynamics in former river channels : prediction testing using a hydroelectric construction. Environmental Management, 18, 223-234.

BOVE G., DI CIACCIO A., 1994. A user-oriented overview of multiway methods and software. Computational Statistics and Data Analysis, 18, 15-37.

CARREL G., BARTHELEMY D., AUDA Y., CHESSEL D., 1986. Approche graphique de l'analyse en composantes principales normée : utilisation en hydrobiologie. Acta CEcologica, Ecologia Generalis, 7, 2, 189-203.

CARREL G., PONT D., RIVIER B., 1995. Variabilité temporelle des peuplements piscicoles dans la section médiane du Bas-Rhône. Bull. Fr. Pêche Piscic., 337/338/339, 101-111.

CENTOFANTI M., CHESSEL D., DOLÉDEC S., 1989. Stabilité d'une structure spatiale et compromis d'une analyse statistique multi-tableaux : Application à la physico-chimie d'un lac réservoir. Revue des Sciences de l'Eau, 2, 1, 71-93.

CHANGEUX T., 1995. Structure du peuplement piscicole à l'échelle d'un grand bassin européen : organisation longitudinale, influence de la pente et tendances régionales. Bull. Fr. Pêche Piscic., 337/338/339, 63-74.

CHESSEL D., MERCIER P., 1993. Couplage de triplets statistiques et liaisons espècesenvironnement. In LEBRETON J.D. et ASSELAIN B., Biométrie et Environnement, 15-44, Masson, Paris.

CHESSEL D., HANAFI M., 1996. Analyses de la co-inertie de $K$ nuages de points. Revue de Statistique Appliquée, 44, 35-60. 
CHESSEL D., SIMIER M., HANAFI M., 1996. STATICO : STATIS et Co-inertie. Documentation thématique ADE-4 "Analyses multivariées et expression graphique des données environnementales ", Vol. 5, section 8, Université Lyon 1, 41 p. (ftp://pbil.univ-lyon1.fr/pub/mac/ADE/ADE4/DocThemPDF/Thema58.pdf).

COSTIL K., CLEMENT B., 1996. Relationship between freshwater gastropods and plant communities reflecting various trophic levels. Hydrobiologia, 321, 7-16.

DEGIORGI F., 1994. Etude de l'organisation spatiale de l'ichtyofaune lacustre. Prospection multisaisonnière de 6 plans d'eau de l'Est de la France à l'aide de filets verticaux. Thèse de l'Université de Franche-Comté, 207 p. + annexes.

DEGIORGI F., GRANDMOTTET J.P., 1993. Relations entre la topographie aquatique et l'organisation spatiale de l'ichtyofaune lacustre: définition des modalités spatiales d'une stratégie de prélèvements reproductible. Bull. Fr. Pêche Piscic., 329, 199-220.

DOLÉDEC S., 1988. Les analyses multi-tableaux en écologie factorielle. II - Stratification longitudinale de l'Ardèche à partir des descripteurs physico-chimiques. Acta CEcologica, CEcologia Generalis, 9, 119-135.

DOLÉDEC S., CHESSEL D., 1987. Rythmes saisonniers et composantes stationnelles en milieu aquatique. I - Description d'un plan d'observations complet par projection de variables. Acta CEcologica, CEcologia Generalis, 8, 3, 403-426.

DOLÉDEC S., CHESSEL D., 1989. Rythmes saisonniers et composantes stationnelles en milieu aquatique. II - Prise en compte et élimination d'effets dans un tableau faunistique. Acta CEcologica, CEcologia Generalis, 10, 3, 207-232.

DOLÉDEC S., CHESSEL D., 1991. Recent developments in linear ordination methods for environmental sciences. Advances in Ecology, India, 1, 133-155.

DOLÉDEC S., CHESSEL D., 1994. Co-inertia analysis : an alternative method for studying species-environment relationships. Freshwater Biology, 31, 277-294.

DOLÉDEC S., DESSAIX J., TACHET H., 1996. Changes within the Upper Rhône river macrobenthic communities after the completion of three hydroelectric schemes: anthropogenic effects or natural change? Archiv für Hydrobiologie, 136, 19-40.

ESCOFIER B., PAGÉS J., 1994. Multiple factor analysis (AFMULT package). Computational Statistics and Data Analysis, 18, 121-140.

ESCOUFIER Y., 1973. Le traitement des variables vectorielles. Biometrics, 29, 750-760.

ESCOUFIER Y., 1987. The duality diagram : a means of better practical applications. In LEGENDRE P. and LEGENDRE L., Development in numerical ecology, 139-156, Springer Verlag, Berlin.

FOUCART T., 1978. Sur les suites de tableaux de contingence indexés par le temps. Statistique et Analyse des données, 2, 67-84.

FOUCART T., 1983. Une nouvelle approche de la méthode STATIS. Revue de Statistique Appliquée, 31, 61-75.

FRANQUET E., DOLÉDEC S., CHESSEL D., 1995. Using multivariate analyses for separating spatial and temporal effects within species-environment relationships. Hydrobiologia, 300/301, 425-431.

GRASMÜCK N., HAURY J., LÉGLIZE L., MULLER S., 1995. Assessment of the bio-indicator capacity of aquatic macrophytes using multivariate analysis. Hydrobiologia, 300/301, 115-122.

HAURY J., BAGLINIĖRE J.L., CASSOU A.I., MAISSE G., 1995. Analysis of spatial and temporal organization in a salmonid brook in relation to physical factors and macrophytic vegetation. Hydrobiologia, 300-301, 269-277.

HERREIRA-SILVEIRA J.A., 1994. Spatial heterogeneity and seasonal patterns in a tropical coastal lagoon. Journal of Coastal Research, 10, 3, 738-746.

HILL M.O., 1973. Reciprocal averaging : an eigenvector method of ordination. Journal of Ecology, 61, 237-249. 
IZAGUIRRE I., VINOCUR A., 1994. Typology of shallow lakes of the Salado River basin, Argentina, based on phytoplancton communities. Hydrobiologia, 277, 49-62.

JAFFRENOU P.A., 1978. Sur l'analyse des familles finies de variables vectorielles. Bases algébriques et application à la description stastistique. Thèse de 3ème cycle, Université Claude Bernard Lyon I, $97 \mathrm{p}$.

KROONENBERG P.M., 1989. The analysis of multiple tables in factorial ecology. III - Threemode principal component analysis : " analyse triadique complète ". Acta CEcologica, CEcologia Generalis, 10, 3, 245-256.

LAIR N., SARGOS D., 1993. A 10 years study at four sites of the middle course of the River Loire. 1 - Patterns of change in hydrological, physical and chemical variables in relation to algal biomass. Hydroécologie Appliquée, 5, 1-27.

LAVIT CH., 1988. Analyse conjointe de tableaux quantitatifs. Masson, Paris, $240 \mathrm{p}$.

LAVIT CH., ESCOUFIER Y., SABATIER R., TRAISSAC P., 1994. The ACT STATIS method. Computational Statistics and Data Analysis, 18, 97-119.

LEIBOVICI B., 1993. Facteurs à mesures répétées et analyses factorielles : application à un suivi épidémiologique. Thèse de Doctorat, Université Montpellier II, $282 \mathrm{p}$.

LETOURNEUR Y., CHABANET P., 1994. Variations spatio-temporelles de l'ichtyofaune de platiers récifaux à la Réunion. Cybium, 18, 1, 25-38.

L'HERMIER DES PLANTES H., 1976. Structuration des tableaux à trois indices de la statistique. Théorie et applications d'une méthode d'analyse conjointe. Thèse de 3ème cycle, USTL, Montpellier, $98 \mathrm{p}$.

MEES J., FOCKEDEY N., HAMERLYNCK O., 1995. Comparative study of the hyper benthos of three European estuaries. Hydrobiologia, 311, 153-174.

MUSSO J.J., PREVOT G., LÉGIER P., PLAYOUST C., 1991. Contribution à la connaissance des cours d'eaux méditerranéens de basse altitude : le réseau hydrographique de l'ARC Bouches-du-Rhône, France. Etat des peuplements en référence aux perturbations anthropiques. Annales de Limnologie, 27, 75-85.

PEGAZ-MAUCET D., 1980. Impact d'une perturbation d'origine organique sur la dérive des macro-invertébrés benthiques d'un cours d'eau. Comparaison avec le benthos. Thèse de 3ème cycle, Université Lyon 1, $130 \mathrm{p}$.

PENAZ M., JURAJADA P., ROUX A.L., OLIVIER J.M., 1995. 0+ fish assemblages in a sector of the Rhône river influenced by the Brégnier-Cordon hydroelectric scheme. Regulated Rivers : Research and Management, 10, 363-372.

POIZAT G., PONT D., 1996. Multi-scale approach to species-habitat relationships : juvenile fish in a large river section. Freshwater Biology, 36, 611-622.

SIMIER M., BLANC L., PELLEGRIN F., NANDRIS D., 1999. Approche simultanée de $K$ couples de tableaux : Application à l'étude des relations pathologie végétale-environnement. Revue de Statistique Appliquée, (sous presse).

THIOULOUSE J., CHESSEL D., 1987. Les analyses multi-tableaux en écologie factorielle. I - De la typologie d'état à la typologie de fonctionnement par l'analyse triadique. Acta CEcologica, CEcologia Generalis, 8, 4, 463-480.

THIOULOUSE J., CHESSEL D., 1992. A method for reciprocal scaling of species tolerance and sample diversity. Ecology, 73, 2, 670-680.

THIOULOUSE J., CHESSEL D., DOLÉDEC S., OLIVIER J.M., 1997. ADE-4 : a multivariate analysis and graphical display software. Statistics and Computing, 7, 1, 75-83.

VERNEAUX J., 1973. Cours d'eau de Franche-Comté Massif du Jura. Recherches écologiques sur le réseau hydrographique du Doubs. Essai de biotypologie. Thèse d'État, Besançon, $257 \mathrm{p}$.

WILLIAMS E.J., 1952. Use of scores for the analysis of association in contingency tables. Biometrika, 39, 274-289. 